\title{
Vortex formation mechanism within fuel streams in laminar nonpremixed jet flames
}

\author{
Min Suk Cha ${ }^{1}$, Jin Woo Son ${ }^{2}$, Sung Hwan Yoon ${ }^{1}$, Hung Truyen Luong ${ }^{2}$, \\ Deanna A. Lacoste ${ }^{1}$, Chae Hoon Sohn ${ }^{2}$ \\ ${ }^{1}$ King Abdullah University of Science and Technology (KAUST) \\ Clean Combustion Research Center (CCRC), Physical Science and Engineering Division (PSE) \\ Thuwal 23955-6900, Saudi Arabia \\ ${ }^{2}$ Department of Mechanical Engineering \\ Sejong University \\ Seoul 05006, Republic of Korea \\ Corresponding Authors \\ General: Min Suk Cha; min.cha@kaust.edu.sa \\ Numerical simulation: Chae Hoon Sohn; chsohn@sejong.ac.kr
}

\begin{abstract}
A vortical structure occurring at the fuel stream in laminar nonpremixed jet flames was recently found and shown to have both a fluid-dynamic impact on the flow field and a possible influence on the flame stability and soot formation. We designed a systematic experiment and numerical simulation to investigate the physical mechanisms of this recirculation phenomenon in a coflow system. We hypothesized that a negative buoyancy, caused by the fuel jet being heavier than the ambient air, may play a significant role in the recirculation. Therefore, we experimentally varied the density of the fuel jet using a binary mixture of methane and n-butane, and tested the density of the coflow oxidizer by replacing nitrogen with carbon dioxide. Several fuel jet velocities, flame temperatures, and nozzle diameters were also studied to thoroughly investigate all parameters that might possibly affect the recirculation. As a result, we found that our modified Richardson number, which is based on the cold density difference between the fuel and the coflow, the flame length, and the jet momentum flux, explained the physical mechanism of the recirculation well, with $\mathrm{Ri} \sim 60$ being the critical value for formation of the recirculation. The negative buoyancy was the primary driving force behind the recirculation, while the jet momentum mitigated its formation.
\end{abstract}

Keywords: nonpremixed flame, negative buoyancy, recirculation, Richardson number 


\section{Introduction}

Laminar nonpremixed coflow jet flames have been studied extensively in soot investigations [1]-[3] due to their relatively simple geometry. They also have been served as a research platform for buoyancy-driven instabilities including flame flickering [4], [5] and lifted flame oscillation [6].

Recently, a recirculating flow pattern was found in the fuel stream near the nozzle exit in laminar nonpremixed jet flames [7]. Such a recirculation in the fuel core is expected to redirect a flow and to accumulate heat, thus, influence the near-nozzle velocity field and the temperature profile along the stream are heavily affected. Thus, the main conclusion of the previous study [7] was that the domain (inlet boundary condition) for a numerical study must be carefully selected in order to capture the effect of the recirculating zone on the related flame characteristics. Because the temperature and flow residence time are important factors for soot formation in laminar nonpremixed jet flames, a recirculation near the nozzle should also affect the smoke point, which is a measure of a soot propensity [8]. A recent study showed the influence of a recirculating structure on aromatic species formation [9], implying that the secondary effect of fluid motion on soot formation only occurs when the fuel used is heavy. Interestingly, nonpremixed jet flames of ethane were numerically predicted to have a recirculating structure very near the nozzle exit when the ambient pressure was higher than $10 \mathrm{~atm}$ [10], which was conceived as a reason behind the experimentally observed increase in soot formation when pressure increased [11].

In addition, the recirculating flow may enhance the flame stability under high-Reynoldsnumber $(\mathrm{Re})$ conditions by generating a low velocity region - no need to introduce a physical bluffbody for flame stabilization. As shown in our previous work [7], for increased Re with n-butane jets, the vortical structure became larger and more complicated, appearing to hold the entire flame. This recirculation may also create flow instability, due to interactions among the various vortical structures occurring in nonpremixed jet flames [12]. Recently, it was published that oscillating laminar lifted flames [13] were also related to a vortex formation caused by negative buoyancy in a fuel core between a nozzle and a lifted flame base. That vortex slowed down the unburned velocity, such that the lifted flame showed a periodic oscillation. 
The recirculating flow has only been observed in propane and n-butane jets; no vortical structure has been found in either methane or ethane jets [7]. Therefore, we hypothesized that the recirculation was a flow pattern intrinsic to fuel streams heavier than the ambient air, due to their negative buoyancy [7]. We suggested that the positive Richardson number, $\mathrm{Ri}=\Delta \rho g d /\left(\rho_{\mathrm{f}} u_{\mathrm{j}}^{2}\right)>0$, which is based on the difference in density between a fuel and the ambient air $\left(\Delta \rho=\rho_{\mathrm{f}}-\rho_{\mathrm{a}}\right)$, where $\rho_{\mathrm{f}}$ is the fuel-stream density, $\rho_{\mathrm{a}}$ is the ambient stream density, $d$ is the nozzle diameter, and $u_{\mathrm{j}}$ is the mean velocity of the fuel at the nozzle exit, is a necessary condition for the recirculation [7].

To validate our hypothesis, we designed an extended systematic experiment. First, we used a binary mixture of methane and n-butane to investigate the influence of fuel density on the transition from a non-recirculating to a recirculating fuel stream. Second, we varied the jet velocity, particularly near the velocity at which the vortex formed, in order to examine the effect of jet momentum. Third, to confirm the effect of positive buoyancy caused by the difference in density between burned and unburned gas, we also varied the flame temperature by changing the oxygen mole fraction in an ambient coflow. Finally, we examined the effect of ambient density by replacing nitrogen with carbon dioxide. To support the experimental results, we performed a numerical simulation.

\section{Experiment and numerical simulation}

The experimental apparatus consisted of the same coflow burner (inner diameter of the fuel nozzle, $d=10.8 \mathrm{~mm}$ ), flow control system, and measurement systems as used in our previous study [7]. The flow fields were visualized by illuminating titanium dioxide $\left(\mathrm{TiO}_{2}\right)$ seeding particles $(\sim 0.3$ $\mu \mathrm{m})$ with a vertical sheet beam from a continuous-wave diode laser (Coherent Inc, Genesis 532-2000 S OPSL, at $532 \mathrm{~nm}$ ). Images of the elastically scattered laser beam, including flame luminosity, were recorded on a digital camera (Cannon, 50D) with a $105 \mathrm{~mm} \mathrm{f} / 2.8$ macro lens (Sigma, DG MACRO), which magnified the small area of interest. To capture the pathlines of the seeding particles, the shutter speed ranged from $1 / 8 \mathrm{~s}$ to $1 / 30 \mathrm{~s}$, depending on the situation. 
Mixtures of methane and n-butane were used to experimentally simulate various fuel densities (represented by the molar mass), such that the molar mass $\left(M_{\mathrm{f}}\right)$ of the mixture was within the range $16.0\left(M_{\mathrm{CH} 4}\right) \leq \mathrm{M}_{\mathrm{f}} \leq 58.1\left(\mathrm{M}_{\mathrm{C} 4 \mathrm{H} 10}\right) \mathrm{g} / \mathrm{mol}$. Air was used as the primary coflow gas. To test the effects of flame temperature on the vortex formation, the coflow air was replaced by an oxygen-enriched nitrogen mixture. Another coflow mixture of oxygen and carbon dioxide (mole fraction of $\mathrm{O}_{2}, X_{\mathrm{O} 2}=$ 0.3) was tested in order to investigate the influence of ambient density. The dependence of the vortex formation on the mean jet velocity $\left(u_{\mathrm{j}}\right)$ was also examined. For all the tested conditions, the coflow velocity $\left(u_{\mathrm{co}}\right)$ was set to $6.2 \mathrm{~cm} / \mathrm{s}$. Mass flow controllers (Brooks Instrument, 0260) maintained the flow rate of each gas.

To compare the experimental results and capture the flow characteristics in detail, a numerical simulation was performed using a general purpose CFD code, FLUENT [14]. Figure 1 shows the computational geometry and grids for the coflow burner. The inlet location of the coflow was set to 10 $\mathrm{mm}$ below the fuel nozzle tip, and the diameter and height of the coflow confinement cylinder were modified to $60 \mathrm{~mm}$ and $120 \mathrm{~mm}$, respectively. Following the main conclusion of the previous study [7], the inlet location of the fuel stream was set to $70 \mathrm{~mm}$ below the nozzle tip in order to capture a realistic velocity profile at the nozzle exit. Based on a grid dependency check, 12,500 quadrilateral elements were assigned to the computational domain. A comparison of the solution with that from 25,000 grids showed less than a $0.5 \%$ difference in terms of temperature and velocity at any location. 


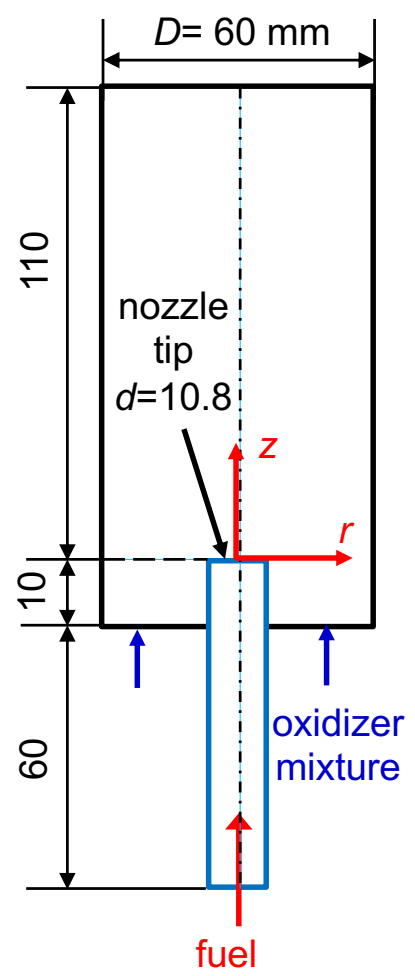

(a) geometry

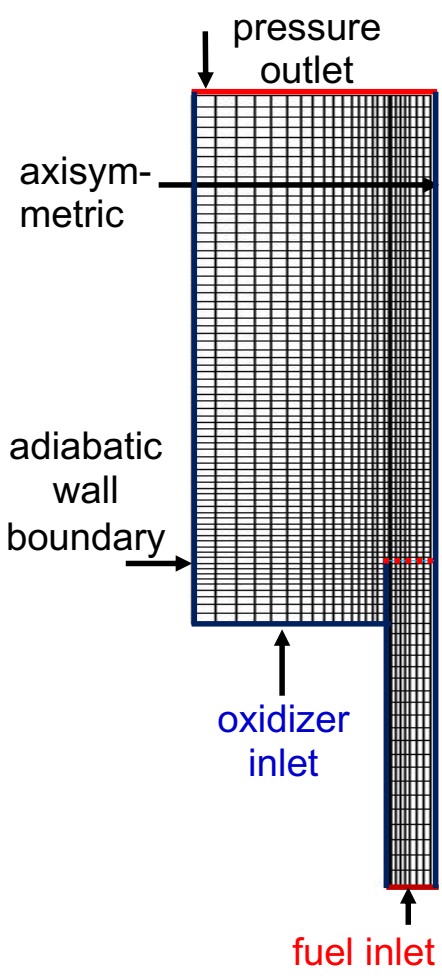

(b) computational grid

Fig. 1. Computational domain and grids of the coflow burner.

A laminar reacting flow was simulated within the domain by solving the steady-state twodimensional governing equations of the continuity, momentum, energy, and species equations simultaneously with the appropriate numerical schemes [15]. The previous study [7] found that onestep global reactions predicted the detailed flow characteristics in fuel streams reasonably well for both methane and propane flames; in terms of the radial and axial profiles of the axial velocity at $z=1 \mathrm{~mm}$ and $r=0$, respectively, both the simulation and the experiment showed negligible quantitative differences [7]. This indicates that, as long as the heat release and reaction rate are properly modeled as a function of the local equivalence ratio, a near-nozzle cold flow with a small nonpremixed laminar jet flame can be predicted precisely regardless of the rigor of the chemistry set adopted.

To predict the structure of flames produced by the experimental fuel mixture considered here, the detailed chemistry of methane and n-butane mixtures, a model of soot and its precursors (polycyclic aromatic hydrocarbons), and the radiative heat transfer must be taken into account. However, the main motivation for the simulation conducted here was to compare the near-nozzle flow field and to support 
the experiment in a qualitative way by conducting a parametric study. Therefore, in this study, the effects of the experimental fuel mixture were simulated by a numerical fuel, circumventing the aforementioned requirements in a cost-effective way. The numerical fuel was defined as having the same properties as methane, but the molar mass was varied artificially in the governing equations. Thus, the isolated effects of fuel density $\left(\rho_{\mathrm{f}}=M_{\mathrm{f}} P / R T\right)$ on the vortex formation could be examined, which would be impossible experimentally.

For the numerical fuel, the one-step global reaction of methane (with an equivalence ratio dependent on the heat release and reaction rate) proposed by Fernández-Tarrazo et al. [16] was used:

$$
\mathrm{CH}_{4}+2 \mathrm{O}_{2} \rightarrow \mathrm{CO}_{2}+2 \mathrm{H}_{2} \mathrm{O}
$$

To predict the adiabatic flame temperature with the one-step global reaction, the heat release per unit mole of methane $(Q)$ was modeled as a function of the equivalence ratio [16]. Here, the equivalence ratio $(\phi)$ was defined based on the mixture fraction $(Z)$ in order to accommodate the nonpremixed nature of the present study:

$$
\phi=4 \frac{Y_{C H 4, j}}{Y_{O 2, c o}} \frac{Z}{1-Z}
$$

where $Y_{\mathrm{CH} 4, \mathrm{j}}$ and $Y_{\mathrm{O} 2 \text {,co }}$ represent the mass fractions of methane and oxygen in their respective streams. The heat release was defined as below depending on the equivalence ratio:

$$
\begin{aligned}
& \phi \leq 1: Q / Q_{o}=1 \\
& \phi \geq 1: Q / Q_{o}=1-\alpha(\phi-1),
\end{aligned}
$$

where $Q_{\mathrm{o}}=802.4 \mathrm{~kJ} / \mathrm{mol}$ and $\alpha=0.21$ for methane [16]. A reaction rate $(k)$ of the above equation can be expressed in the Arrhenius form as [16] 


$$
k=A_{k} \cdot e^{-E_{a} / R T\left(T_{a} / T_{a o}\right)} C_{C H 4} C_{O 2},
$$

where $C_{i}$ denotes the concentration of a species $i, T_{a}$ denotes the activation temperature, and $T_{a o}$ denotes the reference activation temperature. The pre-exponential factor $\left(A_{k}\right)$, the activation energy $\left(E_{a}\right)$, and the gas constant $(R)$ were given as $6.9 \times 10^{11} \mathrm{~m}^{3} /(\mathrm{kmol} \cdot \mathrm{s}), 1.32 \times 10^{11} \mathrm{~J} / \mathrm{kmol}$, and $8,314 \mathrm{~J} /(\mathrm{kmol} \cdot \mathrm{K})$,

respectively [16]. The ratio $T_{a} / T_{a o}$ adjusts the value of $k$ to model the effect of the equivalence ratio via the relations provided below with various domains of $\phi[16]$ :

$$
\begin{array}{ll}
\phi \leq 0.64: & T_{a} / T_{a o}=1+8.250(\phi-0.64)^{2}, \\
0.64 \leq \phi \leq 1.07: & T_{a} / T_{a o}=1, \\
\phi \geq 1.07: & T_{a} / T_{a o}=1+1.443(\phi-1.07)^{2} .
\end{array}
$$

A parabolic velocity profile was applied at the fuel inlet boundary, while the coflow jet velocity $\left(u_{\mathrm{co}}=6.2 \mathrm{~cm} / \mathrm{s}\right.$ ) was uniformly set at the oxidizer inlet. The temperature at the inlets was $300 \mathrm{~K}$. Adiabatic and impermeable conditions were applied to the peripheral boundary, and an atmospheric pressure condition was applied at the axial outlet. The compositions of an oxygen-enriched and a carbon-dioxide-diluted oxygen coflow condition were considered. The detailed experimental and numerical conditions are summarized in Table 1.

Table 1. Experimental and numerical test conditions. In the experiment, the fuel molar mass was varied by changing the mixture ratio of $\mathrm{CH}_{4} / \mathrm{n}-\mathrm{C}_{4} \mathrm{H}_{10}$. In the numerical simulation, the fuel molar mass was artificially changed in the governing equations while maintaining the other properties the same as methane. The coflow velocity was fixed at $u_{\mathrm{co}}=6.2 \mathrm{~cm} / \mathrm{s}$.

Tested domain

\begin{tabular}{ccccc} 
Examined & Varied key & & & \\
\cline { 2 - 4 } effect & parameter & Fuel molar mass & Coflow $\mathrm{O}_{2}$ mole & Jet velocity \\
& $M_{\mathrm{f}}$ & fraction $\left(X_{\mathrm{O} 2}\right)$ and molar & $u_{\mathrm{j}}$ \\
& {$[\mathrm{g} / \mathrm{mol}]$} & mass $\left(M_{\mathrm{a}}[\mathrm{g} / \mathrm{mol}]\right)$ & {$[\mathrm{cm} / \mathrm{s}]$} \\
\hline
\end{tabular}




\begin{tabular}{|c|c|c|c|c|}
\hline $\begin{array}{l}\text { Fuel density } \\
\text { (Section 3.1) }\end{array}$ & $\begin{array}{c}{ }^{1} \text { Mixing ratio of } \\
\mathrm{CH}_{4} \text { and } \mathrm{n}-\mathrm{C}_{4} \mathrm{H}_{10} \\
{ }^{2} \text { Artificially } \\
\text { changed } M_{\mathrm{f}}\end{array}$ & $16<M_{\mathrm{f}}<58.1$ & \multirow{2}{*}{$\underset{\mathrm{Air}}{X_{\mathrm{O} 2}=0.21, M_{\mathrm{a}}=28.8}$} & 8.90 \\
\hline $\begin{array}{l}\text { Jet velocity } \\
\text { (Section 3.2) }\end{array}$ & $u_{\mathrm{j}}$ & $\begin{array}{l}34.6 \\
37.5 \\
46.2\end{array}$ & & $\begin{array}{l}4.45 \\
17.8 \\
22.5\end{array}$ \\
\hline $\begin{array}{l}\text { Flame } \\
\text { temperature } \\
\text { (Section 3.3) }\end{array}$ & $\begin{array}{c}X_{\mathrm{O} 2} \\
\text { in the coflow }\end{array}$ & $\begin{array}{l}31.6 \\
34.6\end{array}$ & $\begin{array}{c}\text { in } \mathrm{N}_{2} \text { balance } \\
X_{\mathrm{O} 2}=0.21, M_{\mathrm{a}}=28.8 \\
X_{\mathrm{O} 2}=0.25, M_{\mathrm{a}}=29.0 \\
X_{\mathrm{O} 2}=0.30, M_{\mathrm{a}}=29.2 \\
X_{\mathrm{O} 2}=0.33, M_{\mathrm{a}}=29.3\end{array}$ & \multirow{2}{*}{8.90} \\
\hline $\begin{array}{c}\text { Coflow density } \\
\text { (Section 3.4) }\end{array}$ & $\begin{array}{c}\mathrm{CO}_{2} \text { replaces } \mathrm{N}_{2} \\
\text { in the coflow }\end{array}$ & $40.3<M_{\mathrm{f}}<48.4$ & $\begin{array}{c}\text { in } \mathrm{CO}_{2} \text { balance } \\
X_{\mathrm{O} 2}=0.30, M_{\mathrm{a}}=40.4\end{array}$ & \\
\hline
\end{tabular}

\section{Results and discussion}

\subsection{Effect of fuel density}

First, we investigated the influence of fuel density on the formation of the vortex. Figure 2 compares the experimentally obtained flow pathlines and the numerically calculated streamlines superimposed on the temperature profiles (pseudo color code) for selected fuel molar masses, where $u_{\mathrm{j}}=8.9 \mathrm{~cm} / \mathrm{s}$ and $X_{\mathrm{O} 2}=0.21$ in nitrogen (simulated air). When the fuel density was equal to that of the coflow (Fig. 2a and e), no recirculating zone was found. As the fuel density became heavier, we identified a small recirculating zone at $M_{\mathrm{f}}=31.6$ in the experiment (no recirculation at $\left.M_{\mathrm{f}}=30.4\right)($ Fig. 2b) and at $M_{\mathrm{f}}=34.6$ in the simulation (Fig. $2 \mathrm{~g}$ ). From this comparison, we can clearly attribute the recirculation in the fuel stream to the increased fuel density, since the simulated recirculation was solely an effect of the numerical fuel's molecular mass. In addition, unlike the previous study [7], we kept the same fuel jet velocity while varying the fuel density; therefore, the observed recirculation was not caused by a difference between the velocities of the fuel jet and the entrained ambient flow. 


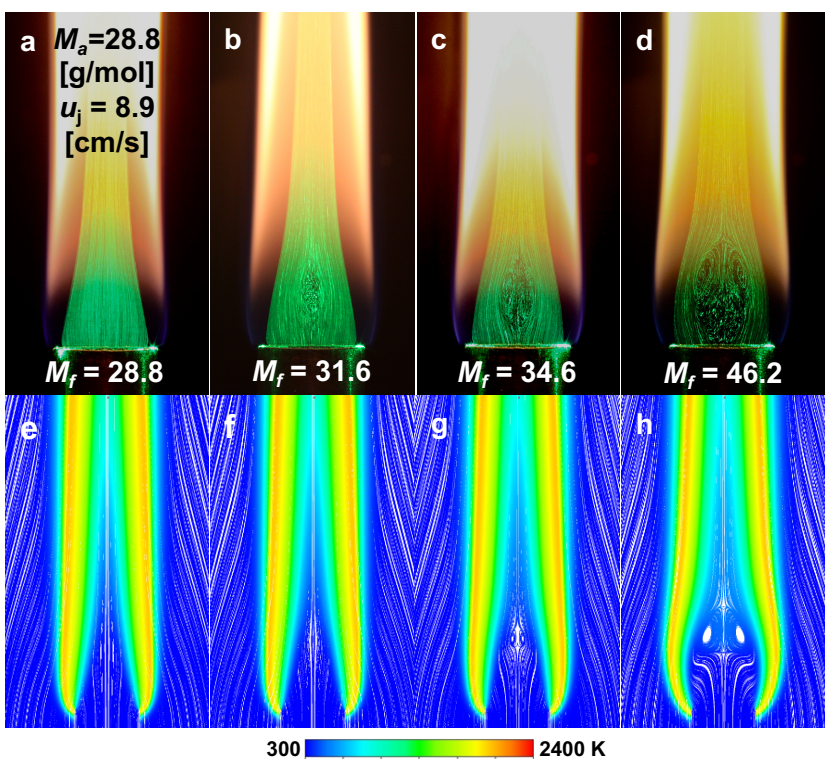

Fig. 2. Flow characteristics for selected fuel molar masses showing experimental (a-d: pathlines) and numerical results (e-h: streamlines and temperature); $u_{\mathrm{j}}=8.9 \mathrm{~cm} / \mathrm{s}, M_{\mathrm{a}}=28.8 \mathrm{~g} / \mathrm{mol}$ (air), and $u_{\mathrm{co}}=6.2 \mathrm{~cm} / \mathrm{s}$.

We expected this quantitative discrepancy between the experimental and numerical results, partly because the physical properties of the experimental fuel mixtures and the numerical fuel were different, and partly because we simplified chemical reaction model. Nevertheless, the simulation reasonably predicted the molecular mass at the onset of recirculation, exhibiting around $10 \%$ difference, and reproduced well the experimentally observed recirculation with increased $M_{\mathrm{f}}$. Once the recirculation formed, both the experimental and numerical results illustrated that the recirculation grew as the fuel density increased. 

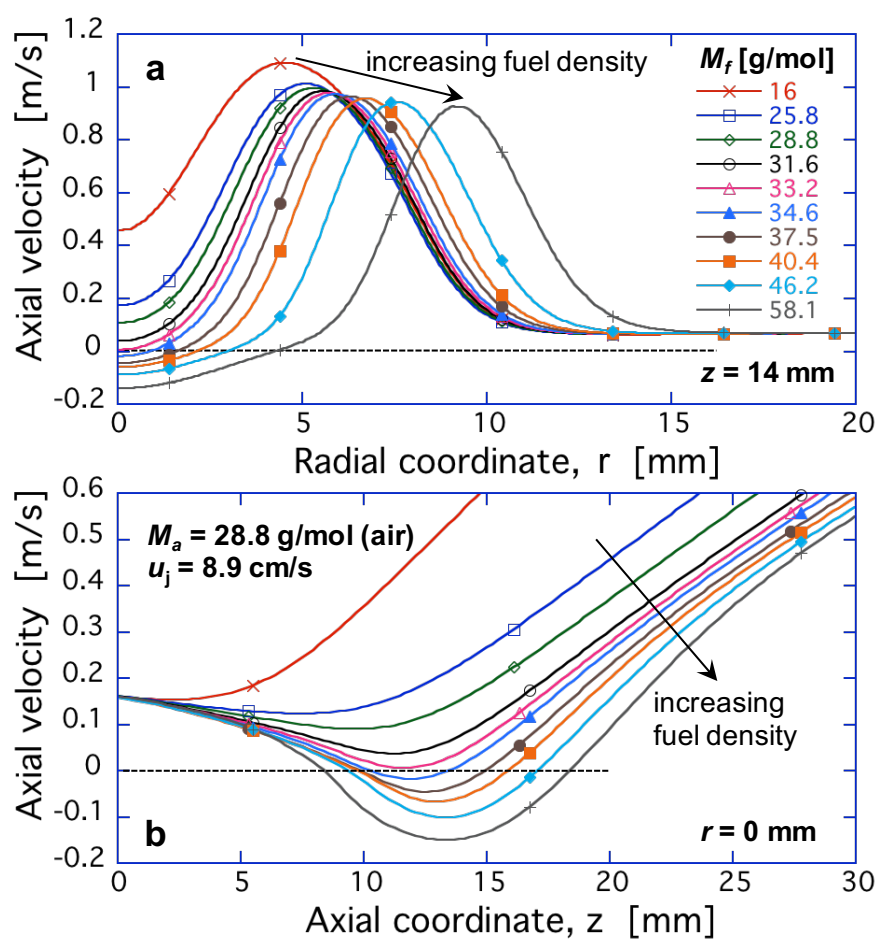

Fig. 3. Numerical results from the radial (a: at $z=14 \mathrm{~mm}$ ) and axial (b: at $r=0$ ) profiles of the axial velocity for various fuel molar masses; $u_{\mathrm{j}}=8.9 \mathrm{~cm} / \mathrm{s}, M_{\mathrm{a}}=28.8 \mathrm{~g} / \mathrm{mol}$ (air), and $u_{\mathrm{co}}=6.2 \mathrm{~cm} / \mathrm{s}$.

The axial and radial profiles of the axial velocity obtained from the numerical results (Fig. 3) show that, as the fuel density increased, a flow region with negative axial velocity appeared, indicating the formation of a recirculating zone. This negative buoyancy, which acted on the fuel core by pulling the fuel stream down toward the nozzle, became stronger with increased fuel density. At the same time, the radial location of the flame (represented by the peak axial velocity in Fig. 3a; $z=14 \mathrm{~mm}$ represents the near-center of the recirculation) was pushed further away from the jet center, reducing the influence of the high velocity flow caused by the flame on the fuel core. These two effects, the negative buoyancy and the changed radial location of the flame, acted together on the fuel core, facilitating the formation of the recirculation in the central region. Figure $3 \mathrm{~b}$ clearly shows the deceleration of the axial velocity with increased fuel density. We found that $M_{\mathrm{f}}=33.2 \mathrm{~g} / \mathrm{mol}$ was the critical molar mass of the numerical fuel for the recirculation, beyond which a negative axial velocity was found along the jet axis.

\subsection{Effect of jet velocity}

Because changing the jet velocity also changes the jet momentum, one of the key forces in this 
recirculation phenomenon as it appears in $\mathrm{Ri}$, we investigated various jet velocities $\left(u_{\mathrm{j}}=4.45,17.8\right.$, and $22.5 \mathrm{~cm} / \mathrm{s}$ ) at $M_{\mathrm{f}}=34.6,37.5$, and $46.2 \mathrm{~g} / \mathrm{mol}$ of the fuel mixtures, where both the experiment and the simulation exhibited a recirculation. When the jet velocity was reduced from $8.9 \mathrm{~cm} / \mathrm{s}$ to $4.45 \mathrm{~cm} / \mathrm{s}$, the axial location of the recirculation zone moved down toward the nozzle, exhibiting a smaller recirculation with a lower location than observed when $u_{\mathrm{j}}=8.9 \mathrm{~cm} / \mathrm{s}$ for $M_{\mathrm{f}}=34.6,37.5$, and 46.2 g/mol; see Fig. $4 \mathrm{a}$ and e $(8.9 \mathrm{~cm} / \mathrm{s})$ and Fig. $4 \mathrm{~b}$ and $\mathrm{f}(4.45 \mathrm{~cm} / \mathrm{s})$ for a comparison at $M_{\mathrm{f}}=34.6$.

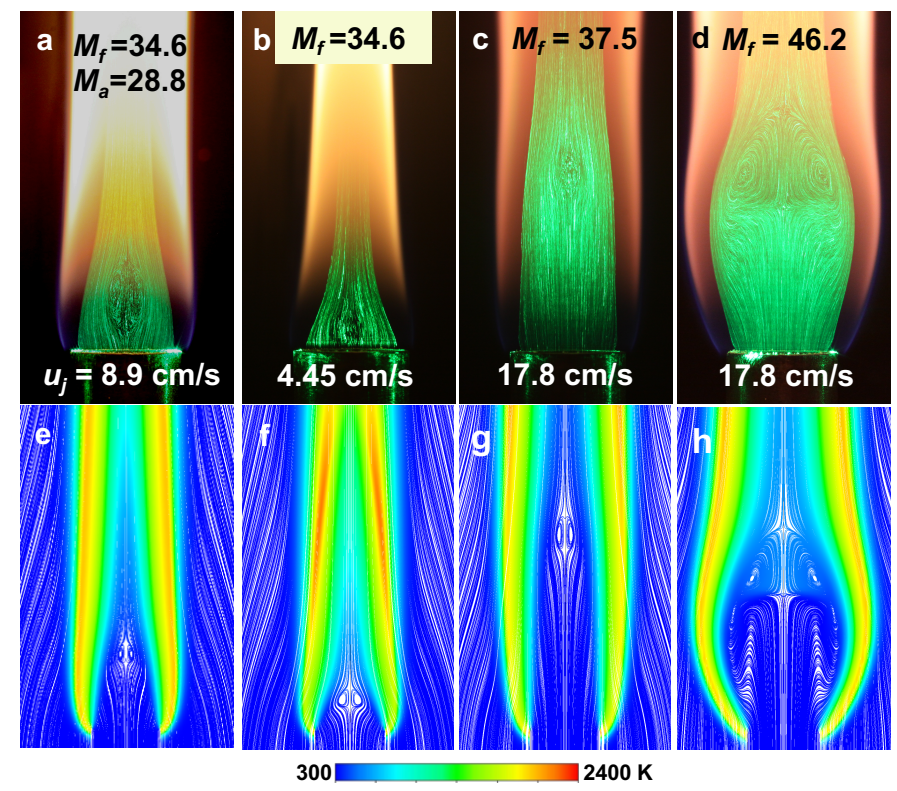

Fig. 4. Selected flow characteristics showing the effect of jet velocity with $M_{a}=28.8 \mathrm{~g} / \mathrm{mol}$ (air): (a, e) $M_{\mathrm{f}}=34.6 \mathrm{~g} / \mathrm{mol}$ and $u_{\mathrm{j}}=8.9 \mathrm{~cm} / \mathrm{s} ;(\mathrm{b}, \mathrm{f}) M_{\mathrm{f}}=34.6 \mathrm{~g} / \mathrm{mol}$ and $u_{\mathrm{j}}=4.45 \mathrm{~cm} / \mathrm{s} ;(\mathrm{c}, \mathrm{g}) M_{\mathrm{f}}=37.5 \mathrm{~g} / \mathrm{mol}$ and $u_{\mathrm{j}}=17.8 \mathrm{~cm} / \mathrm{s} ;$ and $(\mathrm{d}, \mathrm{h}) M_{\mathrm{f}}=46.2 \mathrm{~g} / \mathrm{mol}$ and $u_{\mathrm{j}}=17.8 \mathrm{~cm} / \mathrm{s}$.

However, when the jet velocity was doubled to $17.8 \mathrm{~cm} / \mathrm{s}$, both the experimental and numerical results confirmed that the recirculation zone disappeared at $M_{\mathrm{f}}=34.6$. At $M_{\mathrm{f}}=37.5 \mathrm{~g} / \mathrm{mol}$ (Fig. $4 \mathrm{c}$ and g), the recirculation was still observed with $u_{\mathrm{j}}=17.8 \mathrm{~cm} / \mathrm{s}$, but it had moved away from the nozzle. When the fuel density was further increased to $M_{\mathrm{f}}=46.2$, the recirculation grew larger (Fig. $4 \mathrm{~d}$ and h), consistent with the previous result for n-butane [7]. This indicates that there must be competition between the negative buoyancy acting on the fuel stream and the jet momentum pushing the stream away from the nozzle. Thus, as the fuel density increases, a higher jet velocity is required to eliminate the recirculating zone. 


\subsection{Effect of flame temperature}

Recalling our hypothesized critical $\mathrm{Ri}=\left(\rho_{\mathrm{f}}-\rho_{\mathrm{a}}\right) g d /\left(\rho_{\mathrm{f}} u_{\mathrm{j}}{ }^{2}\right)>0$ for the recirculation in the previous study [7], the recirculation is not supposed to be affected by the positive buoyancy caused by a flame. To study the effect of positive buoyancy on the recirculation, we varied the flame temperature by changing the oxygen mole fraction in the coflow. As shown in Fig. 5, we experimentally considered two fuel molar masses $\left(M_{\mathrm{f}}=31.6\right.$ and $\left.34.6 \mathrm{~g} / \mathrm{mol}\right)$ with $u_{\mathrm{j}}=8.9 \mathrm{~cm} / \mathrm{s}$ at the extended oxygen mole fraction, $X_{\mathrm{O} 2}=0.30$. For $M_{\mathrm{f}}=31.6 \mathrm{~g} / \mathrm{mol}$, which was the near-critical fuel density found experimentally under the test conditions described in Fig. 2, the recirculation zone disappeared at $X_{\mathrm{O} 2}=0.30$ (Fig. 5b). For $M_{\mathrm{f}}=34.6 \mathrm{~g} / \mathrm{mol}$, the recirculation zone was found at $X_{\mathrm{O} 2}=0.30$, though smaller in size (Fig. $5 \mathrm{~d}$ ) than that at $X_{\mathrm{O} 2}=0.21$ (Fig. 5c). The numerical results (shown in Fig. 5e and f) confirmed that the recirculation zone became smaller when, due to increased oxygen content, the flame temperature increased from $\sim 2400 \mathrm{~K}$ to $\sim 3300 \mathrm{~K}$. We controlled for the increased radiation intensity of the soot particles, which was caused by the elevated temperature, by using a laser line optical filter (at $532 \mathrm{~nm}$ ) to capture the clear flow structures.

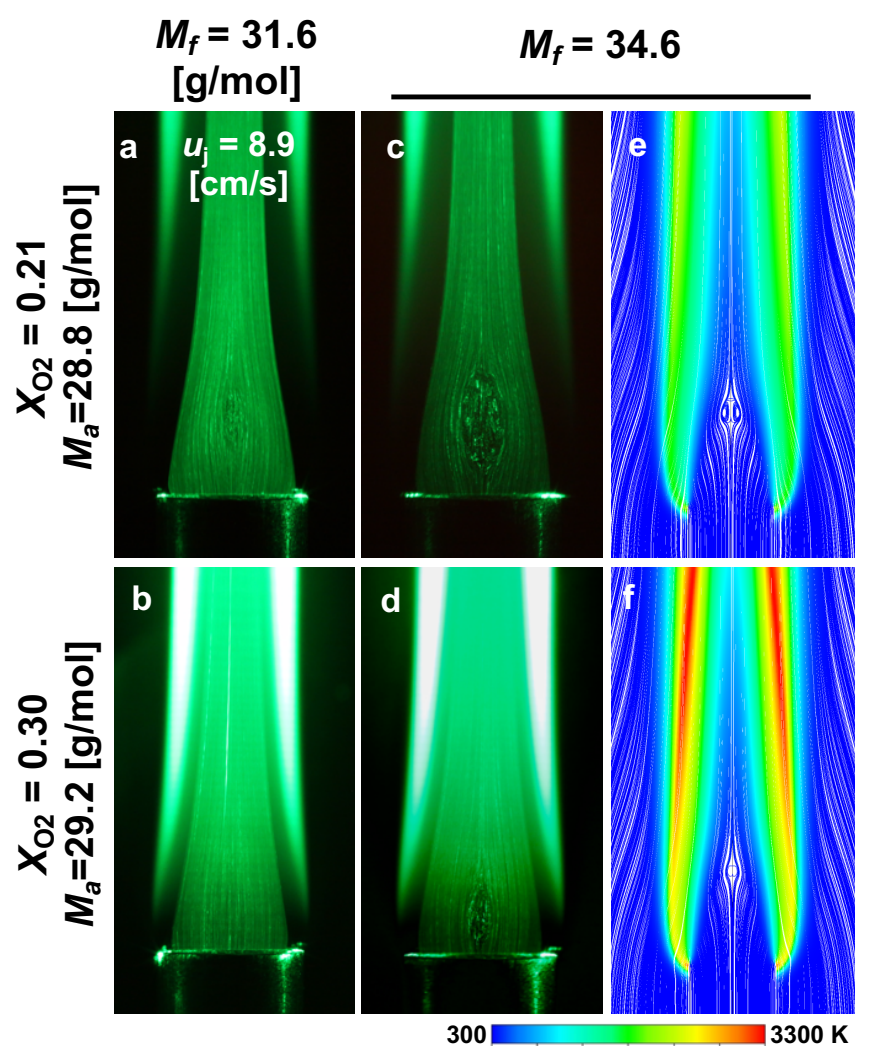

Fig. 5. Effect of flame temperature, controlled by varying the oxygen content in the coflow, on the recirculation. In the upper row, $X_{\mathrm{O} 2}=0.21$ and in the lower row, $X_{\mathrm{O} 2}=0.30$, when $(a, b) M_{\mathrm{f}}=31.6 \mathrm{~g} / \mathrm{mol}$ 
and (c, d: experiment; e, f: numerical) $M_{\mathrm{f}}=34.6 \mathrm{~g} / \mathrm{mol}$ for $u_{\mathrm{j}}=8.9 \mathrm{~cm} / \mathrm{s}$ and $u_{\mathrm{co}}=6.2 \mathrm{~cm} / \mathrm{s}$. The increased radiation from soot particles, caused by elevated flame temperatures, was mitigated in imaging by a $532 \mathrm{~nm}$ laser line filter.

The axial velocity profiles from the numerical results, including the cases of $X_{\mathrm{O} 2}=0.25$ and $X_{\mathrm{O} 2}=0.33$, are plotted in Fig. 6 for $M_{\mathrm{f}}=34.6 \mathrm{~g} / \mathrm{mol}$, since this was the near-critical fuel density for the recirculation in the simulation (Figs. 2 and 3). Near the center of the recirculation at $z=12 \mathrm{~mm}$ (Fig. 6a), we found that the increased oxygen mole fractions increased the axial velocities, which exhibited unaffected radial locations of the flames such that the higher velocity flow due to the flame with increased temperature was still effective at the jet center. Thus, increased flame temperatures suppressed the formation of the recirculation (Fig. 6b). However, when the flame temperature in the simulations increased from $\sim 2400 \mathrm{~K}$ (at $X_{\mathrm{O} 2}=0.21$ ) to $\sim 3700 \mathrm{~K}$ (at $X_{\mathrm{O} 2}=0.33$ ), the local velocity change at $r=0$ and $z=12 \mathrm{~mm}$ was only $2 \mathrm{~cm} / \mathrm{s}$. Therefore, we conclude that the effects of flame temperature on the recirculation were secondary, but should become important near the critical conditions for the formation of the recirculation. In addition to the effects of flame temperature, we note that the molar mass of the ambient at $X_{\mathrm{O} 2}=0.33$ was $1.7 \%$ higher than that of air; this higher molar mass of the ambient also mitigated the recirculation by reducing $\Delta \rho=\rho_{\mathrm{f}}-\rho_{\mathrm{a}}$.
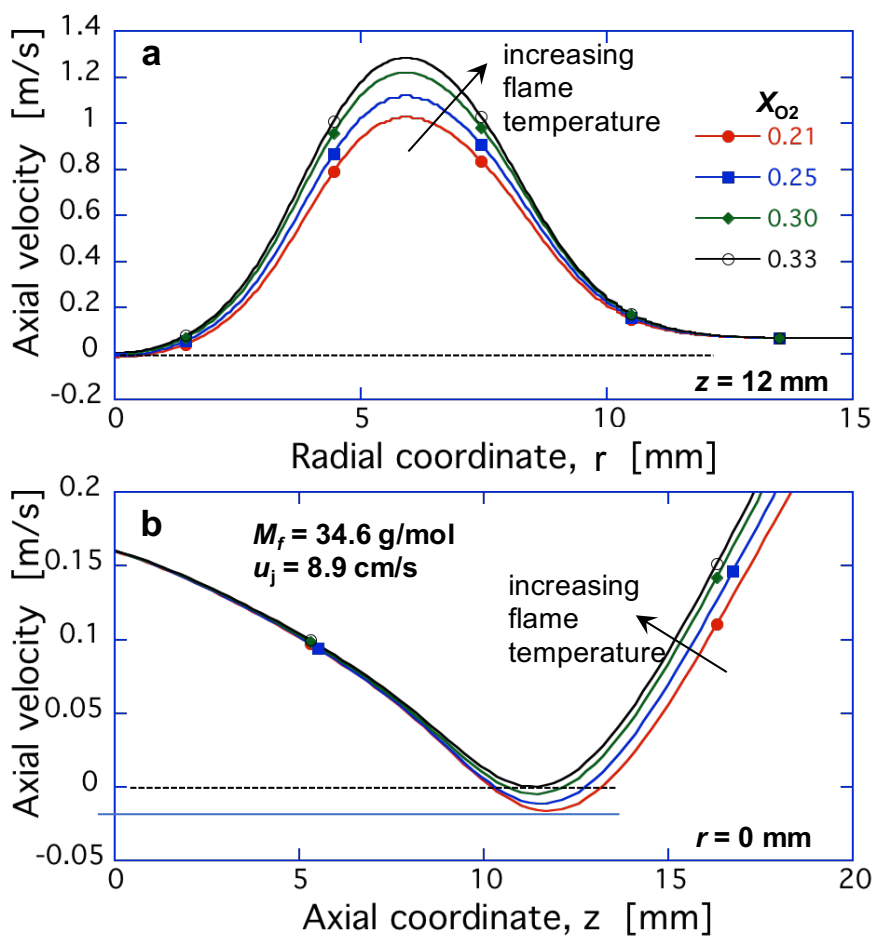

Fig. 6. Numerical results for the radial (a, at $z=12 \mathrm{~mm})$ and axial $(b$, at $r=0)$ profiles of the axial velocity 
with various oxygen mole fractions; $u_{\mathrm{j}}=8.9 \mathrm{~cm} / \mathrm{s}, X_{\mathrm{O} 2}=0.21,0.25,0.30$, and 0.33 , and $u_{\mathrm{co}}=6.2 \mathrm{~cm} / \mathrm{s}$.

\subsection{Effect of coflow density}

Next, we evaluated the effect of $\Delta \rho=\rho_{\mathrm{f}}-\rho_{\mathrm{a}}$ on the recirculation by changing the coflow density. We prepared a mixture of oxygen $\left(X_{\mathrm{O} 2}=0.3\right)$ and carbon dioxide $\left(X_{\mathrm{CO} 2}=0.7\right)$ to increase the ambient density $(40.4 \mathrm{~g} / \mathrm{mol})$, keeping $u_{\mathrm{co}}=6.2 \mathrm{~cm} / \mathrm{s}$. We examined the flow characteristics for various fuel molar masses at $u_{\mathrm{j}}=8.9 \mathrm{~cm} / \mathrm{s}$. The calculated flame temperature with the numerical fuel was $2800 \mathrm{~K}$ and was expected to have a minimal effect on the recirculation, as shown in the previous section.

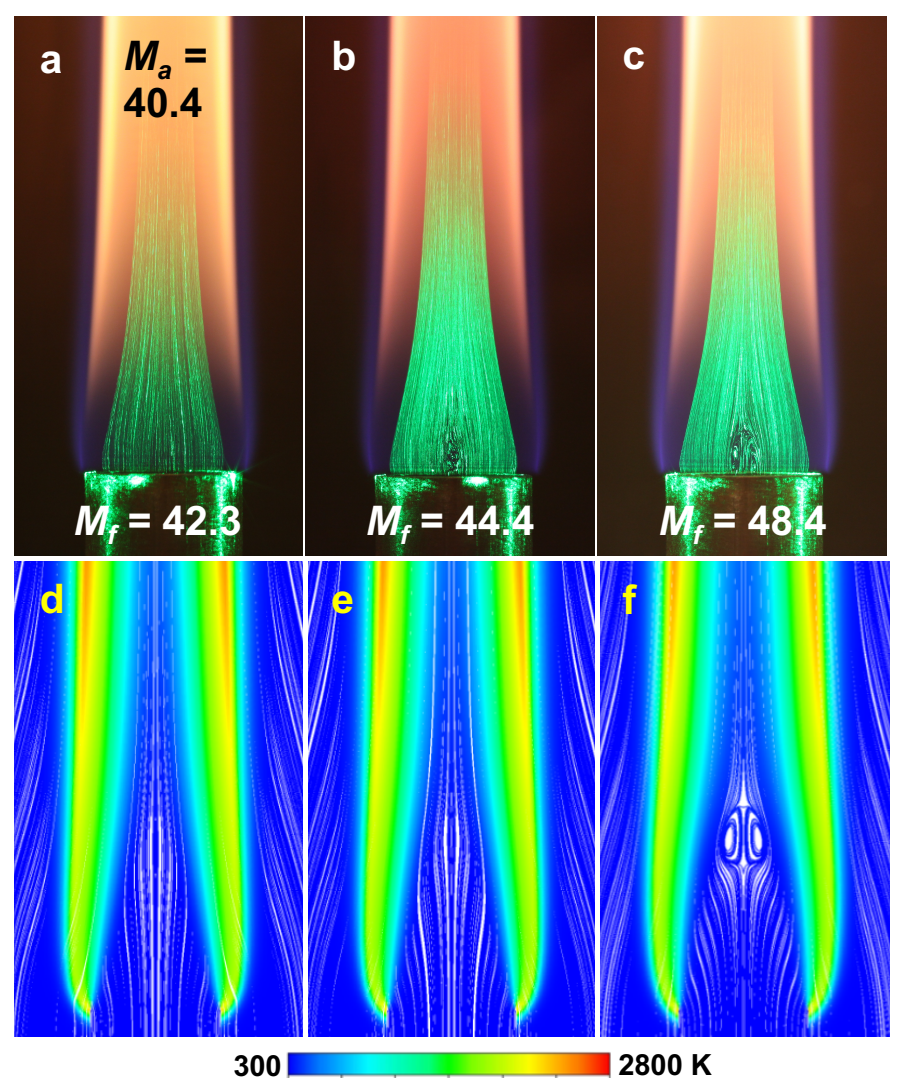

Fig. 7. Recirculation formation due to increased ambient density at $M_{\mathrm{a}}=40.4 \mathrm{~g} / \mathrm{mol}\left(X_{\mathrm{O} 2}=0.3\right.$ and $X_{\mathrm{CO} 2}$ $=0.7$ ) for selected fuel molar masses: $M_{\mathrm{f}}=42.3(\mathrm{a}, \mathrm{d}), 44.3(\mathrm{~b}, \mathrm{e})$, and $48.4 \mathrm{~g} / \mathrm{mol}(\mathrm{c}, \mathrm{f}) ; u_{\mathrm{j}}=8.9 \mathrm{~cm} / \mathrm{s}$ and $u_{\mathrm{co}}=6.2 \mathrm{~cm} / \mathrm{s}$.

Our experiment showed that the recirculation formed at fuel molar masses greater than $M_{\mathrm{f}}=$ $44.4 \mathrm{~g} / \mathrm{mol}$ (Fig. 7a-c), which is a much higher fuel density than found in the flames with coflow air. This finding was intuitively expected because increased ambient density decreases negative buoyancy; 
thus, a higher fuel density should be required to compensate for $\Delta \rho$ and form the recirculation. Again, the simulation qualitatively predicted the trend of vortex formation well, proposing $M_{\mathrm{f}}=46.4 \mathrm{~g} / \mathrm{mol}$ as the critical fuel density (Fig. 8), which is very similar to the experimental value $(44.4 \mathrm{~g} / \mathrm{mol})$, showing a difference of less than $5 \%$. This indicates that $\Delta \rho=\rho_{\mathrm{f}}-\rho_{\mathrm{a}}$ was the key driver behind the observed recirculation because the molar mass of the numerical fuel was the sole parameter varied in the simulation. Figure 8 shows the axial velocity profiles obtained from the simulation, which illustrate the radially expanding flame location and the decreased flow velocity near $r=0$ and $z=14 \mathrm{~mm}$ for high fuel densities, similar to the cases with coflow air (Fig. 3).
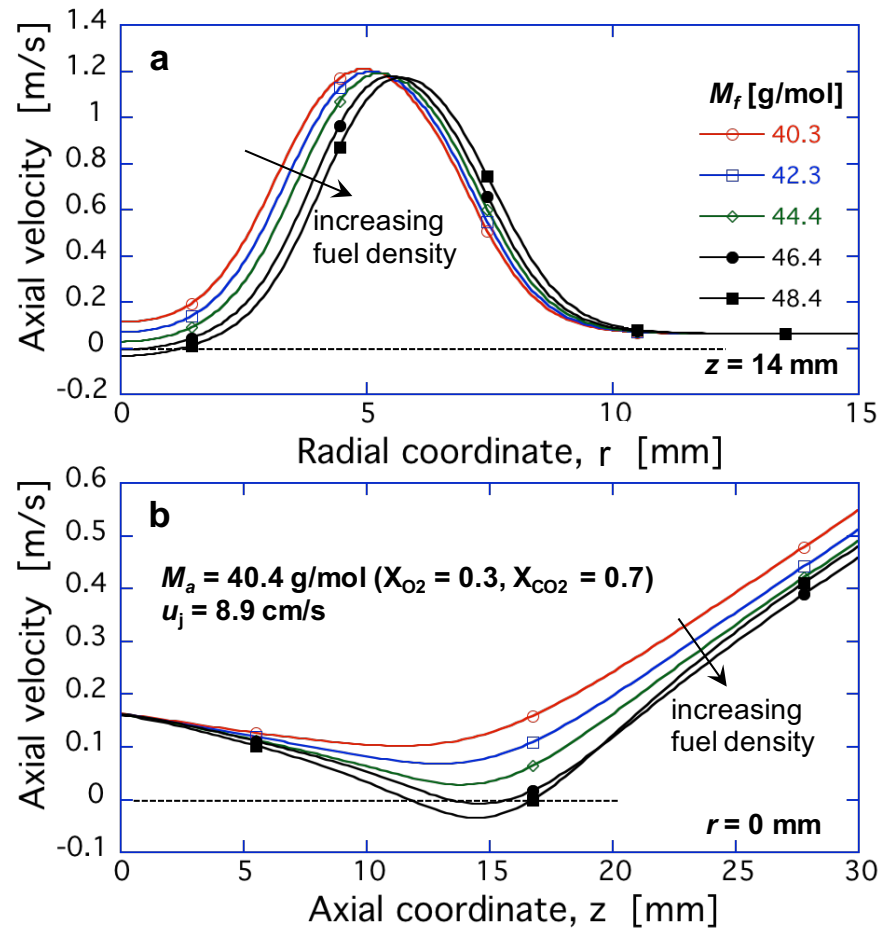

Fig. 8. Numerical results from the radial (a: at $z=14 \mathrm{~mm}$ ) and axial $(\mathrm{b}$ : at $r=0)$ profiles of the axial velocity for various fuel molar masses with increased ambient density at $M_{\mathrm{a}}=40.4 \mathrm{~g} / \mathrm{mol}\left(X_{\mathrm{O} 2}=0.3\right.$, $\left.X_{\mathrm{CO} 2}=0.7\right) ; u_{\mathrm{j}}=8.9 \mathrm{~cm} / \mathrm{s}$ and $u_{\mathrm{co}}=6.2 \mathrm{~cm} / \mathrm{s}$.

\subsection{Validation of the hypothesized physical mechanism}

Negative buoyancy must play a role in the recirculation observed in these fuel streams because it is the only viable source of negative force pulling the fuel stream down. In this regard, we previously hypothesized that $\mathrm{Ri}=\left(\rho_{\mathrm{f}}-\rho_{\mathrm{a}}\right) g d /\left(\rho_{\mathrm{f}} u_{\mathrm{j}}^{2}\right)>0$ was a necessary condition for the recirculation [7]. Major findings in the present results include the following: 1) $\Delta \rho$ has a primary impact on the recirculation; 2) increased jet velocities can prevent the recirculation from forming, indicating that the effect of jet 
momentum prevails over the negative buoyancy with increased jet velocity; and 3) flame temperature is secondary. In this regard, we found that the proposed $\mathrm{Ri}$ was not defined in a way that was compatible with the present system, since the negative buoyancy term is always constant regardless of $u_{\mathrm{j}}$. In fact, a cold fuel region under the influence of negative buoyancy is proportional to the flame length $\left(L_{\mathrm{f}}\right)$; therefore, the nozzle diameter in Ri should be replaced with $L_{\mathrm{f}}$. The laminar flame length can be estimated as a balance between the axial convection time $\left(\sim L_{\mathrm{f}} / u_{\mathrm{j}}\right)$ and the radial diffusion time ( $\sim d^{2} / D$, where $D$ is the mass diffusivity), such that $L_{\mathrm{f}} \sim u_{\mathrm{j}} d^{2} / D$. This is consistent with the results done by Roper et al. [17], [18] showing $L_{\mathrm{f}} \sim Q / D$, where $Q$ is a flow rate of fuel. Therefore, replacing the characteristic length $(d)$ with $L_{\mathrm{f}}$, we define a modified Ri as

$$
\mathrm{Ri} \equiv \frac{\left(\rho_{f}-\rho_{a}\right) g L_{f}}{\rho_{f} u_{j}^{2}} \sim \frac{\left(\rho_{f}-\rho_{a}\right) g u_{j} d^{2} / D}{\rho_{f} u_{j}^{2}}
$$

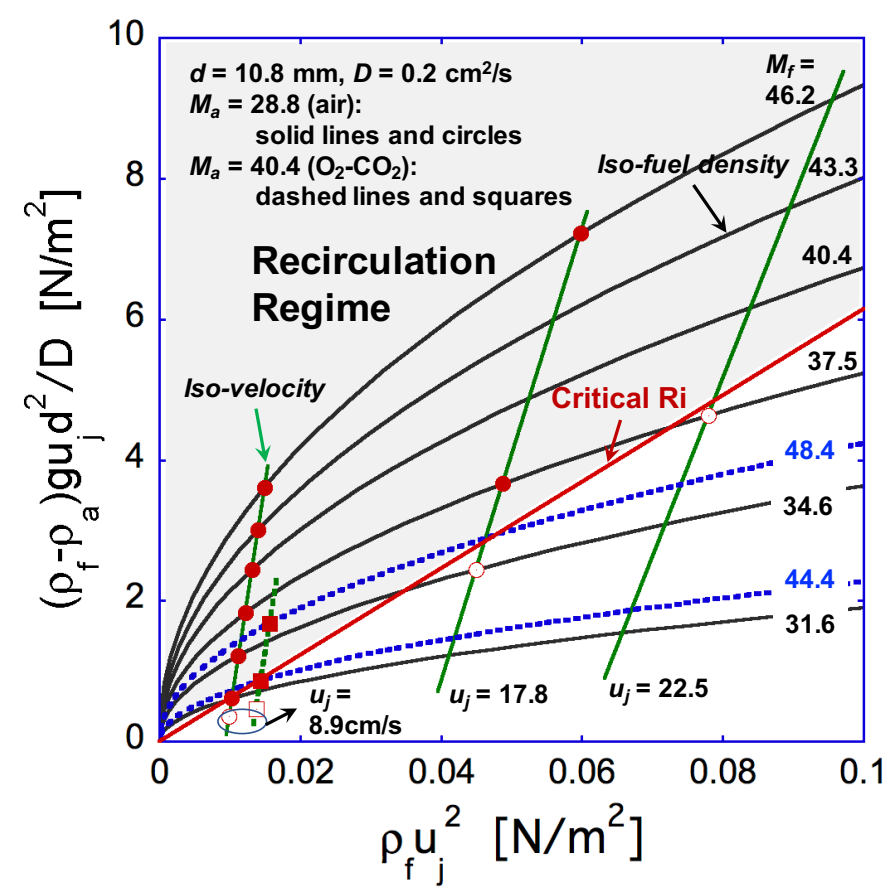

Fig. 9. Domain of the recirculation in terms of jet momentum flux and the negative buoyancy showing that the critical $\mathrm{Ri}$ is $\sim 60$ for the recirculation.

We rearranged all of the experimental test conditions and their results in terms of the jet momentum flux, $\rho_{\mathrm{f}} u_{j}^{2}$, and the negative buoyancy, $\left(\rho_{\mathrm{f}}-\rho_{\mathrm{a}}\right) g u_{j} d^{2} / D$. We used $0.2 \mathrm{~cm}^{2} / \mathrm{s}$ for simplicity, 
since $D=\sim 0.24 \mathrm{~cm}^{2} / \mathrm{s}$ [14] and $D=\sim 0.1 \mathrm{~cm}^{2} / \mathrm{s}$ [19] for methane and n-butane, respectively. While $D$ should be a function of the fuel mixture composition, a representative $D$ is reasonable for predicting the first-order importance of the observed phenomenon. The results are depicted in Fig. 9. We note that only experimental result is shown in Fig. 9. The experimental domain is represented in the figure by iso-fuel density lines and iso-velocity lines. The iso-velocity line of $u_{\mathrm{j}}=8.9 \mathrm{~cm} / \mathrm{s}$ with the circular symbol represents the experiment conducted in Section 3.1, depicting the recirculation formation for $M_{\mathrm{f}} \geq 31.6 \mathrm{~g} / \mathrm{mol}$. Because the negative buoyancy ( $y$-axis of Fig. 9) is proportional to $u_{\mathrm{j}}$ while the momentum flux ( $x$-axis of Fig. 9$)$ is proportional to $u_{\mathrm{j}}{ }^{2}$ along the iso-fuel density lines, by the definition of $\mathrm{Ri}$ above, an increased jet velocity results in different sensitivities of the recirculation to the momentum flux and the negative buoyancy. For low jet velocities, the increases in the negative buoyancy were strongly pronounced compared to the increases in the momentum, resulting in a stronger recirculation. For jet velocities higher than a certain threshold, the increases in the momentum were greater than the increases in buoyancy, suppressing the recirculation. Along the iso-fuel density lines of 34.6 and $37.5 \mathrm{~g} / \mathrm{mol}$, when $u_{\mathrm{j}}$ increased to 17.8 and $22.5 \mathrm{~cm} / \mathrm{s}$, respectively, no recirculation zones were found, as discussed in Section 3.2. This difference in sensitivity explains the larger recirculation as $M_{\mathrm{f}}$ increased to $46.2 \mathrm{~g} / \mathrm{mol}$ from $37.5 \mathrm{~g} / \mathrm{mol}$ at $u_{\mathrm{j}}=17.8 \mathrm{~cm} / \mathrm{s}$ (Fig. $4 \mathrm{c} \rightarrow$ Fig. $4 \mathrm{~d}$ ); for higher values of $M_{\mathrm{f}}$, the increase in buoyancy is greater than the increase in momentum, as shown in Fig. 9.

Assuming that there must be a critical $\mathrm{Ri}$ controlling the recirculation, we drew a line connecting the origin and the condition where $M_{\mathrm{f}}=31.6 \mathrm{~g} / \mathrm{mol}$ and $u_{j}=8.9 \mathrm{~cm} / \mathrm{s}$, representing the nearcritical moment from the iso-velocity experiment (Section 3.1). Other non-recirculating conditions $\left(M_{\mathrm{f}}\right.$ $=34.6 \mathrm{~g} / \mathrm{mol}$ and $u_{\mathrm{j}}=17.8 \mathrm{~cm} / \mathrm{s}$, and $M_{\mathrm{f}}=37.5 \mathrm{~g} / \mathrm{mol}$ and $u_{\mathrm{j}}=22.5 \mathrm{~cm} / \mathrm{s}$ ) occurred well below the recirculation boundary. When we superimposed the result for the heavier coflow density, the fuel density had a reduced effect on the buoyancy; this can be seen in Fig. 9, where the dashed iso-fuel density lines of 44.4 and $48.4 \mathrm{~g} / \mathrm{mol}$ are significantly lower than the lines representing coflow air. More importantly, the critical fuel density at $u_{\mathrm{j}}=8.9 \mathrm{~cm} / \mathrm{s}$ with $M_{\mathrm{a}}=40.4 \mathrm{~g} / \mathrm{mol}$ shows a good agreement 
with the critical $\operatorname{Ri}(\sim 60)$ based on the coflow air.

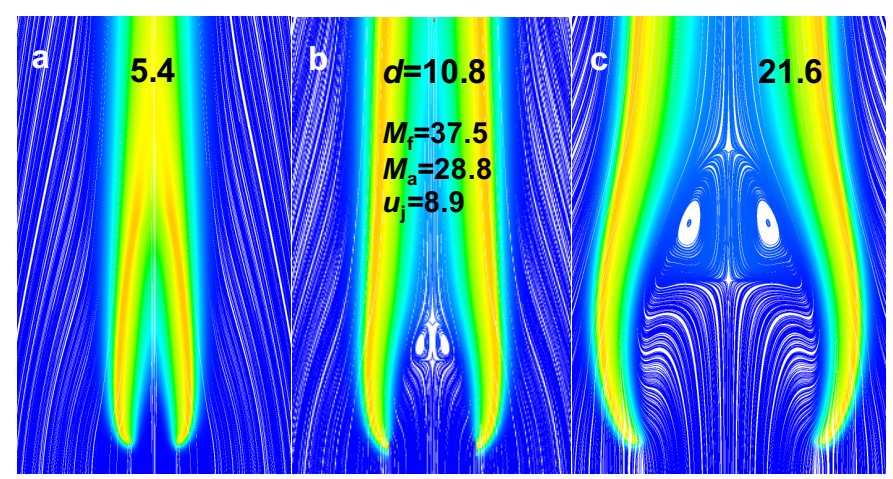

Fig. 10. Calculated streamlines for various nozzle diameters of (a) $d=5.4$, (b) 10.8 , and (c) $21.6 \mathrm{~mm}$; $M_{\mathrm{f}}=37.5 \mathrm{~g} / \mathrm{mol}, M_{\mathrm{a}}=28.8 \mathrm{~g} / \mathrm{mol}, u_{\mathrm{j}}=8.9 \mathrm{~cm} / \mathrm{s}$, and $u_{\mathrm{co}}=6.2 \mathrm{~cm} / \mathrm{s}$.

Finally, we checked the effect of nozzle diameter on the recirculation, since the nozzle diameter appeared in the negative buoyancy term (Eq. 6). In order to isolate the effects of the nozzle diameter, numerical simulations were conducted by varying $d$ and keeping all other conditions fixed $\left(M_{\mathrm{f}}=37.5\right.$ $\mathrm{g} / \mathrm{mol}, M_{\mathrm{a}}=28.8 \mathrm{~g} / \mathrm{mol}, u_{\mathrm{j}}=8.9 \mathrm{~cm} / \mathrm{s}$, and $\left.u_{\mathrm{co}}=6.2 \mathrm{~cm} / \mathrm{s}\right)$. As indicated in Fig. 2, the recirculation appeared in both the experiment and the simulation when $d=10.8 \mathrm{~mm}$. Based on the modified Ri and Fig. 9, the recirculation should disappear when $d$ becomes smaller because the negative buoyancy will be reduced (thus, decreasing the modified $\mathrm{Ri}$ in Eq. 6), and it should become stronger as $d$ becomes larger because the negative buoyancy will increase (thus, increasing the modified Ri). As shown in Fig. 10, a smaller nozzle $(d=5.4 \mathrm{~mm})$ completely eliminated the recirculation (Fig. 10a), whereas a larger nozzle $(d=21.6 \mathrm{~mm})$ created a large recirculation zone (Fig. 10c). This observation suggests that the vortex might affect the smoke point measurement when using a coflow system, depending on the fuel density and diameter of nozzle. However, the widely used Santoro co-flow burner-nominally $11 \mathrm{~mm}$ in diameter-has shown the same measured smoke points as smaller burners (e.g., the Yale burner, which has $4 \mathrm{~mm}$ diameter). In this regard, it would be interesting to simultaneously measure the vortex formation and the smoke point to confirm its effects on the soot propensity.

Thus, we conclude that the recirculation phenomenon is mainly governed by fluid dynamics, while the detailed flame characteristics, such as flame chemistry, are secondary. The critical Ri (using 
our modified definition) based on the experimental result is around 60 , beyond which the recirculation should occur in a laminar flame.

The experimental uncertainty in the critical fuel density, shown in Fig. 9 (iso-velocity line of $u_{\mathrm{j}}$ $=8.9 \mathrm{~cm} / \mathrm{s}$ with coflow air), was less than $4 \%$. In addition, there are many secondary factors not considered in the physical analysis affecting the modified Ri (e.g., mixture diffusivity, flame temperature, a stoichiometric fuel to oxidizer ratio, etc.). This caused uncertainty in the critical Ri, but would not significantly affect the qualitative trends shown in Fig. 9 in the tested range of parameters.

In addition, a recent thesis [10] numerically showed that the recirculation could be observed even with ethane jets, which has a similar molecular weight to air, when using a smaller nozzle $(d \sim 4 \mathrm{~mm})$ under high pressure conditions. That recirculation was not observed until $5 \mathrm{~atm}$, but could be found at $10 \mathrm{~atm}$. A higher entrainment velocity as pressure increased was suggested as a mechanism to generate the vortex. This implies that there might be a general mode of recirculation, which is mostly driven by the difference between the velocities of the fuel jet and the air entrainment caused by positive buoyancy and coflow. This should be an interesting future study for both experimental and numerical approaches.

\section{Concluding remarks}

We conducted a comprehensive and systematic investigation of a recirculating flow structure in the fuel stream of laminar nonpremixed jet flames. To experimentally validate the hypothesis that the recirculation should occur only when the fuel density is greater than that of the ambient coflow, the fuel density was varied by mixing methane and n-butane. In the numerical simulation, only the molar mass of methane was artificially changed to simulate heavier fuels. The effects of the coflow density, fuel jet velocity, and flame temperature were also examined.

We found that the difference in density between the fuel and the coflow gas and the jet velocity were the two most important parameters in determining negative buoyancy, which competed with the jet momentum flux, as represented by the square of the jet velocity. A greater difference between the densities enhanced the recirculation, while a higher jet momentum suppressed it; the effects of flame characteristics, including chemistry, were secondary. 
By redefining the Richardson number based on flame length as $\mathrm{Ri}=\left\{\left(\rho_{\mathrm{f}}-\rho_{\mathrm{a}}\right) g u_{j} d^{2} / D\right\} / \rho_{\mathrm{f}} u_{j}^{2}$, we successfully explained the observed results and extended our hypothesis to a general flame. We proposed $\mathrm{Ri} \sim 60$ as the critical condition for the recirculation onset. Our simple modeling approach predicted the experimental results qualitatively well. An extended simulation with various nozzle sizes successfully supported the validity of our proposed mechanism based on the modified Ri. As small jet diffusion flames are often used in canonical-type experiments studying soot and flame dynamics, including lifted flames and shear layer instability, we hope that the present findings stimulate related fundamental studies of recirculation zones in fuel streams.

\section{Acknowledgments}

The research reported in this publication was supported by funding from King Abdullah University of Science and Technology (KAUST), under Award Number BAS/1/1384-01-01. JWS and CHS were supported by Advanced Research Center Program (NRF-2013R1A5A1073861) through the National Research Foundation of Korea (NRF) grant funded by the Korea government (MSIP) contracted through Advanced Space Propulsion Research Center at Seoul National University.

\section{References}

[1] K.T. Kang, J.Y. Hwang, S.H. Chung, W. Lee, Soot zone structure and sooting limit in diffusion flames: Comparison of counterflow and co-flow flames, Combust. Flame 109 (1997) 266-281.

[2] D.R. Snelling, F. Liu, G.J. Smallwood, Ö.L. Gülder, Determination of the soot absorption function and thermal accommodation coefficient using low-fluence LII in a laminar coflow ethylene diffusion flame, Combust. Flame 136 (2004) 180-190.

[3] M.D. Smooke, R.E. Mitchell, D.E. Keyes, Numerical Solution of Two- Dimensional Axisymmetric Laminar Diffusion Flames, Combust. Sci. Technol. 67 (1989) 85-122.

[4] J. Buckmaster, N. Peters, The infinite candle and its stability - A paradigm for flickering diffusion flames, Symp. (Int.) Combust. 21(1988) 1829-1836. 
[5] T. Yuan, D. Durox, E. Villermaux, An analogue study for flame flickering, Exp. Fluids 17 (1994) 337-349.

[6] S.H. Won, S.H. Chung, M.S. Cha, B.J. Lee, Lifted flame stabilization in developing and developed regions of coflow jets for highly diluted propane, Proc. Combust. Inst. 28 (2000) 2093-2099.

[7] Y. Xiong, M.S. Cha, S.H. Chung, Fuel density effect on near nozzle flow field in small laminar coflow diffusion flames, Proc. Combust. Inst. 35 (2015) 873-880.

[8] L. Li, P.B. Sunderland, An improved method of smoke point normalization, Combust. Sci. Technol. 184 (2012) 829-841.

[9] N.M. Mahmoud, F. Yan, Y. Wang, Effects of fuel inlet boundary condition on aromatic species formation in coflow diffusion flames, J. Energy Inst. (2018) doi.org/10.1016/j.joei.2018.01.007 [10] N.A. Eaves, The effect of reversibility and high pressure on soot formation, $\mathrm{PhD}$ Thesis, University of Toronto, Canada, 2016.

[11] P.M. Mandatori, Ö.L. Gülder, Soot formation in laminar ethane diffusion flames at pressures from 0.2 to 3.3 MPa, Proc. Combust. Inst. 33 (2011) 577-584.

[12] R.W. Davis, E.F. Moore, W.M. Roquemore, L.-D. Chen, V. Vilimpoc, L.P. Goss, Preliminary results of a numerical-experimental study of the dynamic structure of a buoyant jet diffusion flame, Combust. Flame 83 (1991) 263-270.

[13] K.H. Van, J. Park, S.H. Yoon, S.H. Chung, M.S. Cha, Mechanism on oscillating lifted flames in nonpremixed laminar coflow jets, Proc. Combust. Inst. 37 (2019) doi.org/10.1016/j.proci.2018.07.052

[14] ANSYS Inc., ANSYS Fluent Theory Guide, 2017.

[15] T. Poinsot, D. Veynante, Theoretical and Numerical Combustion, R.T. Edwards, Philadelphia, USA, 2001, p. 140.

[16] E.F. Tarrazo, A.L. Sánchez, A. Linan, F.A. Williams, A simple one-step chemistry model for partially premixed hydrocarbon combustion, Combust. Flame 147 (2006) 32-38. 
[17] F.G. Roper, The prediction of laminar jet diffusion flame sizes: Part I. Theoretical model, Combust. Flame 29 (1977) 219-226.

[18] F.G. Roper, C. Smith, A.C. Cunningham, The prediction of laminar jet diffusion flame sizes: Part II. Experimental verification, Combust. Flame 29 (1977) 227-234.

[19] S. Turms, An introduction to combustion: Concept and Application. McGraw-Hill, 2000. 